\title{
TTR
}

Traduction, terminologie, rédaction

\section{Áurea Fernández Rodríguez. Le monde de la bourse en Espagne \\ et en France. Description, glossaire et lexique. La bolsa y su entorno en España y Francia. Descripción, glosario y léxico. Toulouse, Presses Universitaires du Mirail, 2010, 221 p.}

\section{Ana Luna Alonso}

Volume 26, numéro 1, 1er semestre 2013

URI : https://id.erudit.org/iderudit/1036958ar

DOI : https://doi.org/10.7202/1036958ar

Aller au sommaire du numéro

Éditeur(s)

Association canadienne de traductologie

ISSN

0835-8443 (imprimé)

1708-2188 (numérique)

Découvrir la revue

Citer ce compte rendu

Luna Alonso, A. (2013). Compte rendu de [Áurea Fernández Rodríguez. Le monde de la bourse en Espagne et en France. Description, glossaire et lexique. La bolsa y su entorno en España y Francia. Descripción, glosario y léxico. Toulouse, Presses Universitaires du Mirail, 2010, 221 p.] TTR, 26(1), 258-262.

https://doi.org/10.7202/1036958ar d'utilisation que vous pouvez consulter en ligne. 
same challenge that our continued advocacy for visibility finds its most compelling purchase and defense: there is a pressing need to make popular culture an acceptable terrain of investigation. Our discipline's relevance to future generations depends on it.

\section{References}

CRONIN, Michael. (2003). Translation and Globalization. London and New York, Routledge.

CRONIN, Michael. (2006). Translation and Identity. London and New York, Routledge.

Ryan Fraser UNIVERSITY OF OTTAWA

\section{Áurea Fernández Rodríguez. Le monde de la bourse en Espagne et en France. Description, glossaire et lexique. La bolsa y su entorno en España y Francia. Descripción, glosario y léxico. Toulouse, Presses Universitaires du Mirail, 2010, 221 p.}

Cet ouvrage publié par les Presses Universitaires du Mirail (PUM) de l'Université Toulouse-Le Mirail présente les résultats d'une étude de corpus menée par Áurea Fernández Rodríguez, professeure titulaire à la Faculté de traduction de l'Université de Vigo. Ce travail se nourrit d'une certaine expérience de la terminographie, puisque l'auteure a collaboré, entre autres, à l'élaboration du Lexique panlatin de l'énergie éolienne par la Direction de la normalisation terminologique du Bureau de la traduction du Gouvernement du Canada. La présente livraison, qui s'ajoute à la liste de la collection didactique dédiée aux études hispaniques, sous la rubrique "Amphi 7 - Langues. Espagnol», présente selon une perspective comparative les caractéristiques du monde de la Bourse dans les deux espaces concernés, à savoir la France et l'Espagne.

Louvrage s'adresse aux enseignants, chercheurs, professionnels et étudiants de traduction et d'interprétariat de France et d'Espagne, mais s'avère également très utile aux communautés francophones qui s'intéressent aux échanges linguistiques avec l'Espagne et l'Amérique latine. En effet, le texte, rédigé en espagnol, 
constitue un outil particulièrement bien adapté aux étudiants et enseignants du monde universitaire francophone qui privilégient les ouvrages didactiques rédigés en langue originale. Les grandes écoles de commerce, la Licence de Langues étrangères appliquées (LEA) et les instituts de traduction et d'interprétariat français exigent la maîtrise de la terminologie appartenant au domaine des marchés financiers ainsi qu'un bon niveau de connaissances dans ce domaine thématique.

Les marchés des valeurs, que ce soit en Espagne, en France, aux États-Unis ou dans les pays émergents, entraînent la production d'un important volume de textes de référence - analyses financières, rapports annuels, prospectus d'introduction en Bourse, prospectus de fonds, manuels de procédures comptables, etc. - sur le territoire de l'Union européenne, aussi bien en français que dans les autres langues de l'Union. En raison du volume important de transactions des entreprises cotées en Bourse et des intermédiaires, dont les banques, une grande quantité de documents, obligatoires ou optionnels, sont susceptibles d'être traduits. Or, pour cibler des clients d'autres langues et cultures, ces documents doivent être accessibles aux investisseurs étrangers.

Lintérêt de l'ouvrage réside dans son optique pédagogique, soit de former les futurs traducteurs à l'ensemble des spécificités de la traduction économique et financière. L'ouvrage invite d'abord le lecteur à découvrir le monde de la Bourse et des marchés financiers par une présentation théorique des concepts fondamentaux relatifs aux marchés financiers. Il propose ensuite un glossaire de quelque deux cents unités terminologiques accompagnées d'une définition en espagnol et en français. Le contenu du glossaire repose sur la consultation de sources spécialisées et à jour dans le domaine. Enfin, l'ouvrage comprend un lexique espagnol-français ainsi qu'un lexique français-espagnol grâce auxquels les utilisateurs sont à même de repérer rapidement les équivalences des unités terminologiques et phraséologiques recherchées. Les unités qui figurent dans ces lexiques prouvent d'ailleurs que le processus de traduction n'est pas univoque, et qu'il est souvent impossible de traduire mot à mot. En effet, les caractéristiques du discours spécialisé, propre à chaque langue et culture, conditionnent la prise de décisions et le choix de l'unité terminologique adéquate. 
La première partie, à caractère théorique, est sous-divisée en cinq chapitres: les marchés financiers, le système financier espagnol, le système financier français, les grandes références des marchés financiers du monde, c'est-à-dire la Bourse de New York et le Nasdaq et, finalement, des éclaircissements sur l'interaction et l'interdépendance de différents facteurs macroéconomiques (balance commerciale, confiance des consommateurs, inflation, IPC, PNB, PIB, etc.), le prix du pétrole, les taux d'intérêt, la cotation des devises, etc. Ces facteurs sont susceptibles d'avoir un impact sur l'évolution des marchés des valeurs.

Sous forme de synthèse, le premier chapitre présente le parcours historique de la Bourse depuis ses débuts, au XVI siècle, jusqu'à la révolution du numérique, en passant par la révolution commerciale de l'Occident et l'essor de la production industrielle et du capital bancaire, dont l'origine est liée à l'expansion du commerce maritime mondial et au capitalisme commercial campagne-ville. Le tout est accompagné de notes de bas de page qui fournissent des renseignements complémentaires très utiles au lecteur soucieux de mieux connaître l'évolution de ces marchés.

Les explications générales sur le fonctionnement des marchés financiers français et espagnol s'avèrent particulièrement utiles aux fins d'études comparatives. L'auteure fait preuve d'un véritable souci didactique et pédagogique à l'endroit des futurs professionnels de la traduction de textes économiques et financiers. Pour elle, le contrôle des connaissances du domaine est indispensable et préalable à l'acquisition des compétences terminologiques (compétence instrumentale). Les clients qui demandent des services de traduction sont généralement des opérateurs du secteur financier ou encore de petits et moyens épargnants qui souhaitent investir sur les marchés financiers étrangers, mais qui ne maîtrisent pas la langue étrangère ou les enjeux des marchés financiers. Même s'il n'est pas spécialiste des domaines concernés, le traducteur doit, pour répondre aux attentes de destinataires qui, eux, sont spécialistes, être à même de trouver la documentation nécessaire et d'utiliser correctement les outils terminologiques et phraséologiques du domaine en question. Voilà pourquoi, selon l'auteure, la capacité d'utiliser les sources documentaires tient une place centrale dans l'ensemble des compétences à acquérir pour traduire des textes spécialisés en général, et des textes économiques, 
voire financiers, en particulier. En effet, Internet est devenu un outil très utile, voire indispensable, mais il faut savoir chercher, car tout ce qui circule sur Internet n'est pas fiable.

En ce qui concerne les ressources terminologiques sur support papier ou numérique, il faut dire que l'on ne trouve ni sur le marché espagnol de l'édition ni sur le marché français de ressources qui fournissent à la fois la signification des termes et leur traduction en espagnol et en français. Les professeurs et les professionnels de la traduction connaissent bien les ouvrages de référence des domaines bancaires et financiers; ils savent ainsi qu'il n'est pas toujours facile d'obtenir des explications claires sur les concepts de ces domaines dans des langues autres que l'anglais.

Le Dictionnaire des marchés financiers de J. Antoine et M. Capiau-Huart (De Boeck, 2008), l'un des derniers ouvrages parus sur support papier, est un outil multilingue précieux, mais trop cher pour l'étudiant. Il existe d'autres ressources utiles, notamment la base de données terminologiques du Service de traduction de la Commission européenne, IATE (Inter Active Terminology for Europe; http://iate.europa.eu/), réputée d'une grande fiabilité et reconnue comme une autorité en différents domaines. Néanmoins, les définitions que l'on y trouve, souvent tirées de textes traduits, sont moins crédibles. On peut aussi penser au Grand dictionnaire terminologique (GDT; http://gdt.oqlf.gouv. qc.ca/) de l'Office québécois de la langue française, qui contient des fiches relatives à certains concepts des domaines économique et financier, et dont le contenu est présenté principalement en français, et parfois en anglais et en d'autres langues. Cependant, nous n'y trouvons par exemple aucune fiche sur des termes tels que actif toxique, actif pourri et banque poubelle. Ce dernier terme pourrait pourtant s'ajouter à la liste des synonymes fournis pour structure de défaisance. TermiumPlus, la banque de terminologie du gouvernement du Canada (http://www.btb.termiumplus.gc.ca/), est également très pratique, mais s'avère incomplète en ce qui concerne notamment la terminologie relative à la crise financière. À titre d'exemple, les termes crédit à risque, ladrillo et toxic asset en sont absents.

Nous pouvons mentionner d'autres bases de données terminologiques, par exemple, celle des Nations Unies (UNTERM; https://unterm.un.org/), qui offre des solutions 
en anglais, français, espagnol, russe et arabe, ou encore celle du Service de traduction de 1'UNESCO (UNESCOTERM; http:// termweb.unesco.org/), qui englobe l'arabe, le chinois, l'espagnol, le français, l'anglais et le russe. Cependant, comme c'est le cas des autres banques de terminologie mentionnées précédemment, elles ne sont pas spécialisées dans les domaines qui nous intéressent. Enfin, les ressources terminologiques offertes par l'Organisation mondiale du commerce (OMC; WTOTERM; https://wto. sdlproducts.com/multiterm) et le Fonds monétaire international (FMI; http://www.imf.org/external/np/term/fra/index.htm), ne contiennent pas de définitions.

Dans un tel contexte, le glossaire que propose Áurea Fernández Rodríguez ne peut que répondre à un besoin. Celuici, créé à partir d'un corpus de textes parallèles en français et en espagnol portant sur la crise financière, vise à éclairer le sens des termes en discours. Une telle approche permet de voir comment les locuteurs de chaque culture perçoivent la réalité de la crise et comment celle-ci est exprimée par le biais de termes tels que hipotecas basuras, productos tóxicos, turbulencias financieras, grandes quiebras bancarias, crisis del ladrillo, etc. Les termes répertoriés proviennent de la presse de vulgarisation et de la presse spécialisée; des variantes terminologiques de différents registres sont aussi proposées. Le résultat: une ressource fort utile pour l'enseignement de la traduction spécialisée ainsi que pour les professionnels des deux langues concernées.

Ana Luna Alonso UNIVERSIDADE DE VIGO

Edwin Gentzler. Translation and Identity in the Americas: Nerw Directions in Translation Theory. New York, Routledge, 2008, 232 p.

In Translation and Identity in the Americas: New Directions in Translation Theory, author Edwin Gentzler investigates the critical role translation plays in creating, transforming and reflecting the identities of those who live in Central, North and South America. Emphasizing minority and/or marginalized groups 